\title{
Selective Access for Supply Chain Management in the Cloud
}

\author{
Anselme Tueno*, Florian Kerschbaum ${ }^{\dagger}$, Daniel Bernau* Sara Foresti ${ }^{\ddagger}$, \\ *SAP Research, Karlsruhe, Germany - Email: anselme.kemgne.tueno@sap.com, daniel.bernau@sap.com \\ †University of Waterloo, Waterloo, Canada - Email: florian.kerschbaum@uwaterloo.ca \\ ${ }^{\ddagger}$ Università degli Studi di Milano, 26013 Crema, Italy - Email: sara.foresti@unimi.it
}

\begin{abstract}
Object-level tracking along supply chains, enabled by the low-cost and wide availability of Radio Frequency Identification (RFID) technology, permits companies to collect large amounts of data (e.g., time, location, handling) about the goods they produce. Combining the data collected by the different companies along a supply chain can provide considerable advantages to all of them. However, such a sharing sometimes needs to be selective. Indeed, companies may need to keep some information about their business operations secret. In this paper, we propose a solution that enables selective sharing of data collected along a supply chain. Our solution uses the services offered by cloud providers for sharing data among companies, and relies on selective encryption for enforcing access restrictions over such data.
\end{abstract}

Index Terms-Selective encryption, supply chain policy

\section{INTRODUCTION}

Thanks to the wide availability today of Radio Frequency Identification (RFID) technology [13] at limited prices, companies are more and more adopting object-level tracking in their supply chains. Indeed, object-level tracking provides business benefits and is sometimes requested by regulations (e.g., in the pharmaceutical industry). RFID technology provides the means to equip and capture each object with an unique identifier and hence enables companies to easily collect information about it. Data commonly collected in supply chains include time, location, and type of handling (e.g., packing, unpacking, receiving, or shipping). On one hand, combining these data from all the companies along a supply chain (not just predecessor and successor of each phase) enables or improves many economically attractive collaborative applications, including batch recalls [29], counterfeit detection [28], benchmarking and analytics [18]-[20], or estimated arrival forecasts [6]. On the other hand, information collected along the supply chain may be considered sensitive as it allows espionage on the business operations of the involved companies [12], [23]. The need for keeping a subset of the collected data secret, also to (some of) the other companies along the supply chain, represents a major obstacle to wider sharing of object-level tracking data.

In this paper, we propose a solution for enabling companies collaborating in the production process to selectively share object-level tracking data. The proposed solution leverages the services offered by the cloud for data sharing and relies on a Trusted Third Party (TTP) offering a Public Key Infrastructure

\begin{tabular}{l|cccc|}
\multicolumn{1}{c}{} & \multicolumn{1}{c}{$\mathbf{t}_{\mathbf{1}}$} & $\mathbf{t}_{\mathbf{2}}$ & $\mathbf{t}_{\mathbf{3}}$ & $\mathbf{t}_{\mathbf{4}}$ \\
\cline { 2 - 5 } $\mathbf{A}$ & 1 & 1 & 0 & 1 \\
$\mathbf{B}$ & 1 & 0 & 0 & 1 \\
$\mathbf{C}$ & 1 & 0 & 1 & 1 \\
$\mathbf{D}$ & 0 & 0 & 1 & 1 \\
\cline { 2 - 5 } & & &
\end{tabular}

Fig. 1: An example of access matrix

(PKI). Therefore, collected data are stored in a centralized cloud database that all the collaborating companies can access. Since the cloud provider itself may not be authorized for the sensitive content of the collected data, and then also to enforce access restrictions, the proposed approach relies on selective encryption for enforcing the authorization policy regulating access to collected data. Intuitively, each company will encrypt different portions of the collected information using different encryption keys, which are then distributed to the other companies along the chain, in such a way that each company can decrypt all and only the data it is authorized to access.

The reminder of this paper is organized as follows. Section II illustrates how selective encryption can be used to enforce access control restrictions. Section III focuses on the policies that should be used to regulate visibility over objects' data in supply chain scenarios. Section IV introduces our approach, based on selective encryption, for effectively enforcing these visibility policies. Section V summarizes related works. Finally, Section VI presents our conclusions.

\section{SELECTIVE ENCRYPTION FOR ACCESS CONTROL ENFORCEMENT}

Given a relation $r$, defined over relation schema $R\left(a_{1}, \ldots, a_{n}\right)$, the authorization policy regulating access to the tuples in $r$ by the users in the system can be represented through an access matrix AM, having a row for each user $u \in \mathbb{U}$ and a column for each tuple $t \in r$. Cell $A M[u, t]$ in the access matrix has value 1 iff $u$ is authorized to access $t$, it has value 0 otherwise. The set of users who can access a tuple $t$ represents its access control list, acl $(t) \subseteq \mathbb{U}$. Figure 1 illustrates an example of access matrix regulating access to a relation composed of 4 tuples $\left\{t_{1}, \ldots, t_{4}\right\}$ for a set $\mathbb{U}=\{A, B, C, D\}$ of 4 users. As an example, $\operatorname{acl}\left(t_{1}\right)=A B C$. 


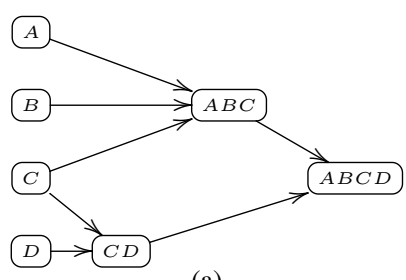

(a)

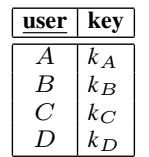

(b)

\begin{tabular}{|c|l|}
\hline tuple & \multicolumn{1}{|c|}{ key } \\
\hline \hline$t_{1}$ & $k_{A B C}$ \\
$t_{2}$ & $k_{A}$ \\
$t_{3}$ & $k_{C D}$ \\
$t_{4}$ & $k_{A B C D}$ \\
\hline
\end{tabular}

(c)

\begin{tabular}{|c|l|l|}
\hline source & \multicolumn{1}{|c|}{ target } & \multicolumn{1}{c|}{ token } \\
\hline \hline$l_{A}$ & $l_{A B C}$ & $k_{A B C} \oplus h\left(k_{A}, l_{A B C}\right)$ \\
$l_{B}$ & $l_{A B C}$ & $k_{A B C} \oplus h\left(k_{B}, l_{A B C}\right)$ \\
$l_{C}$ & $l_{A B C}$ & $k_{A B C} \oplus h\left(k_{C}, l_{A B C}\right)$ \\
$l_{C}$ & $l_{C D}$ & $k_{C D} \oplus h\left(k_{C}, l_{C D}\right)$ \\
$l_{D}$ & $l_{C D}$ & $k_{C D} \oplus h\left(k_{D}, l_{C D}\right)$ \\
$l_{C D}$ & $l_{A B C D}$ & $k_{A B C D} \oplus h\left(k_{C D}, l_{A B C D}\right)$ \\
$l_{A B C}$ & $l_{A B C D}$ & $k_{A B C D} \oplus h\left(k_{A B C}, l_{A B C D}\right)$ \\
\hline
\end{tabular}

(d)

Fig. 2: Encryption policy equivalent to the authorization policy in Figure 1

Selective encryption represents an effective solution for enforcing access control restrictions over data stored at an external, possibly not fully trusted, cloud provider. Indeed, selective encryption guarantees that the data self-enforce access control restrictions. Hence, the enforcement of the authorization policy regulating access to the data requires the intervention neither of the data owner nor of the cloud provider. Intuitively, selective encryption consists in encrypting different tuples with different encryption keys, and in distributing keys in such a way that the key used to protect a tuple is known to all and only the users authorized to it. The adoption of selective encryption requires the definition of an encryption policy equivalent to $A M$. The encryption policy establishes the key(s) to be used to encrypt each tuple $t \in r$ as well as key distribution to users. An encryption policy is equivalent to, and hence correctly enforces, an authorization policy if each user is able to decrypt all and only the tuples she is authorized to access (i.e., $u$ can decrypt $t$ iff $A M[u, t]=1)$.

To translate the authorization policy into an equivalent encryption policy, the solution in [10], [11] defines a different key $k_{u}$ for each user $u \in \mathbb{U}$ and a key $k_{U}$ for each set $U$ of users representing the access control list of a tuple $t \in r$. Each user $u$ is then assigned key $k_{u}$ and each tuple $t \in r$ is encrypted with $k_{U}$, with $U=a c l(t)$. Equivalence between the encryption policy and the authorization policy is guaranteed by using a token-based key derivation technique [1], enabling the derivation of each key $k_{U}$ from any key $k_{u}$ such that $u \in U$. For key derivation, each encryption key $k_{i}$ is associated with a public label $l_{i}$. Token token $_{i, j}=k_{j} \oplus h\left(k_{i}, l_{j}\right)$, with $\oplus$ the bitwise xor operator and $h$ a collusion resistant cryptographic hash function, permits to derive key $k_{j}$ from the knowledge of $k_{i}$ and public label $l_{j}$. Graphically, keys and tokens can be represented in a key derivation graph with a node for each key $k$ and an edge $\left(k_{i}, k_{j}\right)$ for each token token ${ }_{i, j}$. Equivalence to the authorization policy is then guaranteed if there exists a path from $k_{u}$ to $k_{U}$ iff $u \in U$. Indeed, this permits any user $u$ authorized to access a tuple $t$ to derive key $k_{U}$, with $U=a c l(t)$, used to encrypt $t$ starting from the knowledge of her own key $k_{u}$. Figure 2 illustrates an encryption policy equivalent to the authorization policy in Figure 1. Figure 2(a) illustrates the key and token graph, while Figures 2(b-c) summarize the keys assigned to users and the keys used to encrypt tuples, respectively. Figure 2(d) reports publicly available tokens, stored at the cloud provider.

Since the encryption policy should be equivalent to the authorization policy to guarantee its proper enforcement, updates to the authorization policy translate into updates to the corresponding encryption policy. Intuitively, every time a user is granted (revoked, respectively) access to a tuple, it should be (re-)encrypted with a key known to all and only the users in the new access control list. If such a key does not exist, the data owner needs to generate it, together with the set of tokens necessary to enable its derivation. For instance, if user $B$ is granted access to $t_{2}$, then $\operatorname{acl}\left(t_{2}\right)=A B$. To enforce such a policy update, the data owner needs to generate a new encryption key, $k_{A B}$, and the tokens that enable users $A$ and $B$ to derive it. She will then re-encrypt $t_{2}$ with $k_{A B}$. Note that, in some situations, it is not necessary to generate a new key and re-encryption can be avoided. If user $u$ is granted access to a tuple $t$ and no other tuple is encrypted with the same key as $t$, it is sufficient to add a token enabling $u$ to derive the key used for $t$. For instance, if user $A$ is granted access to $t_{3}$, then $\operatorname{acl}\left(t_{3}\right)$ becomes $A C D$. Since $k_{C D}$ is used to protect $t_{3}$ only, the data owner can simply add a token enabling $A$ to derive $k_{C D}$ from $k_{A}$.

\section{SUPPLY Chain}

In this section, we illustrate the problem facing supply chain partners, and describe visibility policies and the access control protocol that will allow to enforce fine-grained access to supply chain data.

\section{A. Problem Definition}

Imagine a set of mobile physical objects $o_{1}, \ldots, o_{m}$ each traversing a (potentially different) subset of players $p_{1}, \ldots, p_{n}$. Each player $p_{i}$ collects information about each object $o_{j}$ it handles (e.g., time, place, type of action, etc.) and stores this information in a central cloud database. Later other players may ask to access an object's data in this database. This scenario is common place in modern supply chains [27]. Figure 3 illustrates an example of a supply chain where a manufacturer $p_{1}$ produces two objects $o_{1}, o_{2}$, collects information $I_{11}, I_{21}$ and ships both objects to distributor $p_{2}$. The distributor collects information $I_{12}, I_{22}$ and ships $o_{1}, o_{2}$ to retailers $p_{3}, p_{4}$ respectively. Later, a supply chain partner may want to access the data collected by another one.

Companies are usually part of many supply chains (even for the same product), and collected data may be stored in a single cloud database. Specifying access control rules for the tuples in this database can be very delicate. Consider the following two examples. Imagine a supplier $p_{2}$ selling a product $o_{2}$ to 


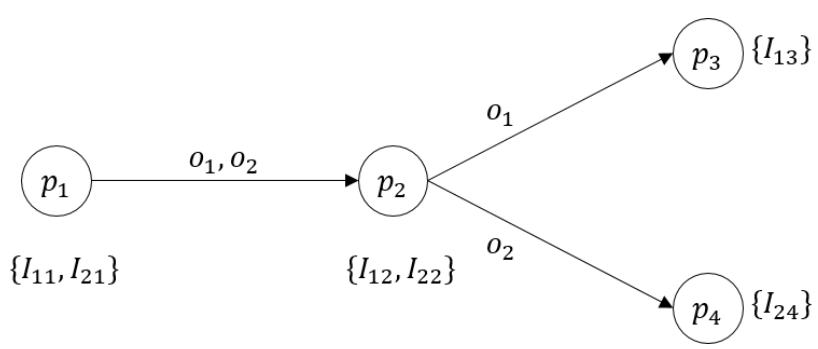

Fig. 3: An example of a supply chain

buyers $p_{3}$ and $p_{4}$. If buyer $p_{3}$ has access to all scheduled orders for $o_{2}$, she can infer the volume of future business with $p_{4}$. This can be very sensitive, in case supplier $p_{2}$ has to cancel some orders due to a temporary capacity reduction (e.g., a machine failure). Buyer $p_{3}$ could then infer whether $p_{4}$ 's orders are treated preferentially. While this decision can be based on local information, in the case of bridging only one supply chain stage, it becomes difficult in case of a tier-2 supplier (i.e., a supplier's supplier). As another example, imagine a supplier $p_{1}$ selling product $o_{1}$ to $p_{2}$ which is then used by $p_{2}$ to produce $o_{2}$. Again, assume that $p_{2}$ later sells object $o_{2}$ to players $p_{3}$ and $p_{4}$. If either buyer $p_{3}$ or $p_{4}$ contacts supplier $p_{1}$ requesting data, $p_{1}$ cannot decide which object was shipped to which buyer. If supplier $p_{1}$ would grant access to all items, buyer $p_{3}$ could infer again the volume of business of $p_{4}$.

A naturally emerging access rule is to share data with partners about shared objects, that is, objects a group of partners have possessed. This implements the important business concept of visibility [21], that is, each partner gains (additional) information about how its (entire) supplies are produced and how its (entire) products are used, but still provides a separation between different supply chains merging at one company. Furthermore it can be easily adopted reciprocally (i.e. "I give you access, if you give me access") providing a fair allocation of cost, risk, and benefits.

We distinguish between downstream and upstream visibility. In downstream visibility a company is allowed to access data associated with its objects shipped to its supply chain partners (at those partners). Upstream visibility is the reverse and a company is allowed to access data associated with objects it has received from its supply chain partners (again at those partners).

\section{B. Visibility Policies}

Visibility policies allow each partner to gain information about how its supplies are produced and how its products are used, but still provides a separation between different supply chains merging at one company. We now review the notion of visibility policies introduced in [21]. To this purpose, we first introduce the trajectory of an object $o_{j}$, and then present the definition of upstream and downstream visibility policies.

Definition 1: Let there be $n$ players $p_{i} \in \mathbb{X}=\left\{p_{1}, \ldots, p_{n}\right\}$. The trajectory $L\left(o_{j}\right)=\left\langle\mathbb{L}_{j}, \mathbb{R}_{j}\right\rangle$ of object $o_{j}$ is modeled as a totally ordered set consisting of elements in the set $\mathbb{L}_{j} \subseteq \mathbb{X}$ and a binary relation $\mathbb{R}_{j} \subseteq \mathbb{L}_{j} \times \mathbb{L}_{j}$. The players represent the spatial domain of the trajectory and the players in $\mathbb{L}_{j}$ are those that have handled (possessed) the object $o_{j}$. Relation $\mathbb{R}_{j}$ models the temporal domain of the trajectory.

Simply speaking, a player $p_{i}$ is ranked lower than a player $p_{i^{\prime}}$ in $L\left(o_{j}\right)$, that is, $\left\langle p_{i}, p_{i^{\prime}}\right\rangle \in \mathbb{R}_{j}$, if $p_{i}$ handled the object $o_{j}$ earlier than $p_{i^{\prime}}$. We write $\sigma\left(p_{i}, L\left(o_{j}\right)\right)$ for a predicate that can be used to compute the rank of player $p_{i}$ in $L\left(o_{j}\right)$ and $\left|\sigma\left(p_{i}, L\left(o_{j}\right)\right)\right|$ for the evaluated rank itself. The predicate $\sigma\left(p_{i}, L\left(o_{j}\right)\right)$ can be seen as a chained proof of the path followed by object $o_{j}$ to player $p_{i}$. This means, each sender adds a proof to the chain that it sent the object to the next receiver. In this case, we see $\left|\sigma\left(p_{i}, L\left(o_{j}\right)\right)\right|$ as the length of the proof $\sigma\left(p_{i}, L\left(o_{j}\right)\right)$ if it is indeed true. We also assume the existence of an external party, the trusted third party, that helps to prove the source of the chain and to compute the rank (Section III-C). Then, $\left|\sigma\left(p_{i}, L\left(o_{j}\right)\right)\right|<\left|\sigma\left(p_{i^{\prime}}, L\left(o_{j}\right)\right)\right|$ iff $\left\langle p_{i}, p_{i^{\prime}}\right\rangle \in \mathbb{R}_{j}$. If player $p_{i}$ did not handle the object $o_{j}$, then $\left|\sigma\left(p_{i}, L\left(o_{j}\right)\right)\right|$ is UNDEFINED and any order relation on the natural numbers, (e.g., both $<$ and $>$ ), should always evaluate to false. We say the least element in $L\left(o_{j}\right)$ is the source of object $o_{j}$ and the top most element is its destination.

In the example in Figure 3, the trajectory of $o_{1}$ is $L\left(o_{1}\right)=$ $\left\langle\left\{p_{1}, p_{2}, p_{3}\right\},\left\{\left(p_{1}, p_{2}\right),\left(p_{2}, p_{3}\right)\right\}\right\rangle$. As the proof of the source is provided by the trusted third party, the rank of $p_{1}, p_{2}, p_{3}, p_{4}$ are respectively $1,2,3$, UNDEFINED.

A player $p_{i}$ is part of multiple supply chains if at least two objects that it handled have been handled by at least one player each - both upstream or downstream - which did not handle both objects. For example distributor $p_{2}$ is in the supply chain of both objects $o_{1}$ and $o_{2}$.

Now assume, player $p_{i}$ is requesting information from player $p_{v}$ (verifier) about object $o_{j}$ stored in the cloud database. Player $p_{v}$ intercepts this request and performs an access control decision. The intercepting component is called a policy enforcement point (PEP) and the information about the request, (e.g., the identity of the requestor $p_{i}$ and the unique identifier of the object $o_{j}$ ) are forwarded to the policy decision point (PDP). The PDP compares the information with the policies in its store and returns its evaluation decision (grant or deny) to the PEP, which will then enforce grant (or deny) access.

Definition 2: An upstream visibility policy grants (or denies) access to $p_{i}$ for $o_{j}$ based on the predicate evaluation

$$
\left|\sigma\left(p_{v}, L\left(o_{j}\right)\right)\right|<\mid \sigma\left(p_{i}, L\left(o_{j}\right) \mid\right. \text {. }
$$

With reference to the example in Figure 3, player $p_{3}$ can be granted access to the information collected at $p_{1}, p_{2}$ only on object $o_{1}$. Similarly, player $p_{4}$ can be granted access to information collected at $p_{1}, p_{2}$ only on object $o_{2}$.

Definition 3: A downstream visibility policy grants (or denies) access to $p_{i}$ for $o_{j}$ based on the predicate evaluation

$$
\left|\sigma\left(p_{i}, L\left(o_{j}\right)\right)\right|<\mid \sigma\left(p_{v}, L\left(o_{j}\right) \mid .\right.
$$


With reference to the example in Figure 3 , player $p_{1}$ can be granted access to the information collected at $p_{2}, p_{3}, p_{4}$ on objects $o_{1}$ and $o_{2}$.

\section{Access Control}

Existing access control models are not scalable when protecting object-level data, because the authorization matrix is too huge. Since the concept of visibility is independent of the players on the trajectory of an object, it can be used to reduce the authorization matrix and simplify its administration. Moreover, it is possible to unify visibility policies with attribute-based access control (ABAC) [24] without necessarily sacrificing simplicity of administration. This unification is important as companies may want to deny access to competitors or allow access to players outside the supply chain, such as auditors [21].

The enforcement of access control restrictions requires a preliminary authentication phase. The authentication phase is a protocol executed between the requestor and the data owner. It is assumed that players are uniquely identifiable and can reliably compute the predicate $\sigma\left(p_{i}, L\left(o_{j}\right)\right)$. Moreover, a trusted third party (TTP) should be available for providing a PKI and RFID tag to players. Finally, it is also assumed that the communication between each player and the TTP is secure and authenticated and that RFID tags have re-writable permanent storage. The access control protocol operates in three steps: initialize, move, and authenticate [21].

Initialize: The trusted third party $(T)$ sends to player $p_{i}$ an RFID tag with identifier $i d$ which contains in its storage the signature $S_{T}\left(i d, p_{i}\right)$.

Move: Player $p_{i}$ wants to move an object to another player $p_{j}$. She appends to the storage on the RFID tag the recipient's identity $\left(p_{j}\right)$ and her signature $S_{p_{i}}\left(i d, p_{j}\right)$.

Authenticate: Let $s_{1}, \ldots, s_{\kappa}$ be the sequence of signatures stored on the RFID tag attached to object $o_{i d}$. The requestor $p_{i}$ sends as the verifiable predicate $\sigma\left(p_{i}, L\left(o_{i d}\right)\right)$ this sequence $s_{1}, \ldots, s_{\kappa}$. The verifier $p_{v}$ verifies that

1) $s_{\kappa}$ is equal to $S p_{i_{\kappa}}\left(i d, p_{i}\right)$.

2) $\forall \lambda \in\{2, \ldots, \kappa-1\}, s_{\lambda}$ is equal to $S_{p_{i_{\lambda}}}\left(i d, p_{i_{\lambda+1}}\right)$.

3) $s_{1}$ is equal to $S_{T}\left(i d, p_{i_{2}}\right)$.

4) $\forall \lambda \in\{1, \ldots, \kappa\}, s_{\lambda}$ is valid signature from $p_{i_{\lambda}}$.

If all checks are successful, then $p_{v}$ evaluates its policies to make the access decision.

Figure 4 provides an example of supply chain operations based on the above protocol. Assume a manufacturer $M$ produces a good. She first starts the Initialize protocol by contacting the trusted third party $T$ and requesting an RFID tag. $T$ chooses a tag for the good with identifier $g$, stores on the tag memory $s_{1}=S_{T}(g, M)$ and sends the RFID tag to $M . M$ reads $\left\langle s_{1}\right\rangle$ from the tag, stores it in its database and attaches the tag to the good $g$. Now $M$ intends to ship $g$ to its distributor $D$. She starts the Move protocol and appends
$s_{2}=S_{M}(g, D)$ to the tag's memory. $M$ can then ship $g$ to $D$. When $D$ receives the good $g$, she reads $\left\langle s_{1}, s_{2}\right\rangle$ from the tag and stores it in her database. Later $D$ may ship $g$ to the retailer $R$. In this Move protocol she appends $s_{3}=S_{D}(g, R)$ to the tag's memory. $R$ reads $\left\langle s_{1}, s_{2}, s_{3}\right\rangle$ from the tag of the received good $g$ and stores it in her database.

Now, assume that $M$ wants to send a downstream request to $R$, (e.g., in order to collect sales data or analyze product returns). $M$ and $R$ run an Authenticate protocol. $M$ reads $\left\langle s_{1}\right\rangle$ from her database and sends it along with $g, M$ to $R$. $R$ 's PEP verifies the data in $\left\langle s_{1}\right\rangle$. It extracts $M, g$, and the number of signatures (1) from $\left\langle s_{1}\right\rangle$. Then it looks up its corresponding predicate $\sigma(R, L(g))=\left\langle s_{1}, s_{2}, s_{3}\right\rangle$ in its database and similarly extracts $R, g$, and the number of signatures (3).

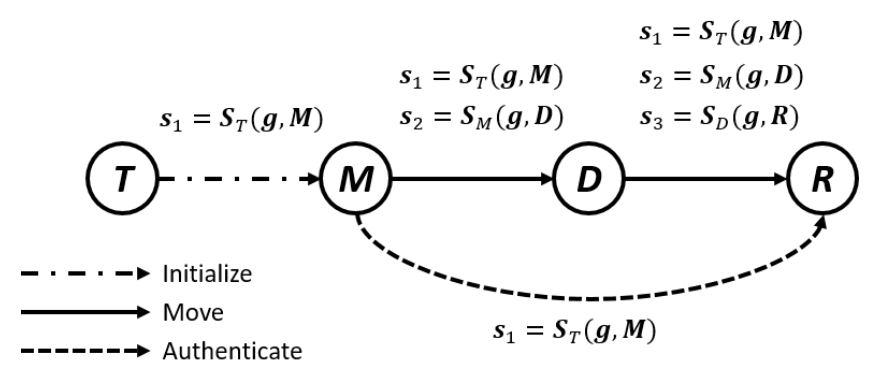

Fig. 4: An example of an object traversing a supply chain

\section{Supply Chain Access Control Enforcement}

In this section, we illustrate how selective encryption (Section II) can be profitably used to enforce supply chain visibility policies (Section III). To properly enforce the visibility policy through selective encryption, each player $p$ chooses her own private key $k_{p}$. Every time an object moves through the supply chain, each player who possesses it collects information about it. This information is represented as a tuple, which is then stored in a database in the cloud to simplify sharing with other players. Since the data collected about objects moving through supply chains are sensitive, tuples are encrypted before being inserted into the cloud database. To this aim, the player collecting the data chooses a unique symmetric encryption key $k_{O}$ (and a corresponding public label $l_{o}$ ) for each object $o$ that she handles, and uses such a key to encrypt the tuple $t$ about $o$. Even if in principle each tuple $t$ generated by player $p$ could be encrypted with her own key $k_{p}$, we use a different key for tuple encryption to keep the role of player's private keys and that of encryption keys separate.

To illustrate how selective encryption can be used to enforce the visibility policy of supply chains, we analyze each of the steps of the access control protocol described in Section III. In the following discussion, we will refer our examples to the simple supply chain and object traversal illustrated in Figure 4. The manufacturer $M$ has private key $k_{M}$, the distributor $D$ has key $k_{D}$, and the retailer $R$ has key $k_{R}$. 


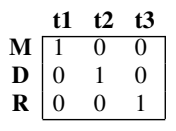

(a)

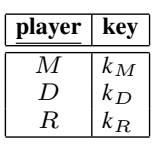

(b)



(c)

\begin{tabular}{|c|l|c|}
\hline source & target & token \\
\hline \hline$l_{M}$ & $l_{1}$ & $k_{1} \oplus h\left(k_{M}, l_{1}\right)$ \\
$l_{D}$ & $l_{2}$ & $k_{2} \oplus h\left(k_{D}, l_{2}\right)$ \\
$l_{R}$ & $l_{3}$ & $k_{3} \oplus h\left(k_{R}, l_{3}\right)$ \\
\hline
\end{tabular}

(d)

Fig. 5: Access matrix and encryption policy subsequently to the move step

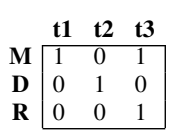

(a)

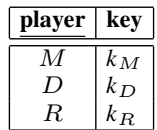

(b)

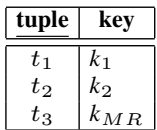

(c)

\begin{tabular}{|c|l|c|}
\hline source & target & token \\
\hline \hline$l_{M}$ & $l_{1}$ & $k_{1} \oplus h\left(k_{M}, l_{1}\right)$ \\
$l_{M}$ & $l_{3}$ & $k_{3} \oplus h\left(k_{M}, l_{3}\right)$ \\
$l_{D}$ & $l_{2}$ & $k_{2} \oplus h\left(k_{D}, l_{2}\right)$ \\
$l_{R}$ & $l_{3}$ & $k_{3} \oplus h\left(k_{R}, l_{3}\right)$ \\
\hline
\end{tabular}

(d)

Fig. 6: Access matrix and encryption policy after authentication of $M$ for $t_{3}$

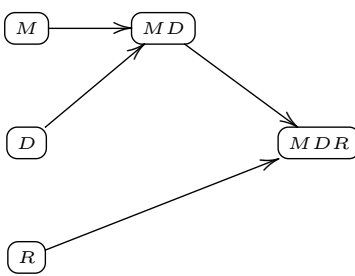

(a)

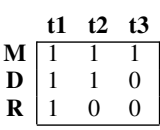

(b)



(c)

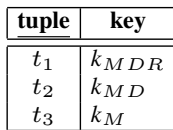

(d)

\begin{tabular}{|c|l|l|}
\hline source & target & \multicolumn{1}{c|}{ token } \\
\hline \hline$l_{M}$ & $l_{M D}$ & $k_{M D} \oplus h\left(k_{M}, l_{M D}\right)$ \\
$l_{D}$ & $l_{M D}$ & $k_{M D} \oplus h\left(k_{D}, l_{M D}\right)$ \\
$l_{M D}$ & $l_{M D R}$ & $k_{M D R} \oplus h\left(k_{M D}, l_{M D R}\right)$ \\
$l_{R}$ & $l_{M D R}$ & $k_{M D R} \oplus h\left(k_{R}, l_{M D R}\right)$ \\
\hline
\end{tabular}

(e)

Fig. 7: An example of key derivation graph, access matrix, and encryption policy

Initialize. At initialization time, a player $p$ requests a set of RFID tags from the trusted third party and possibly starts to create objects. With reference to our example, after receiving the RFID tag from $T$, the manufacturer $M$ creates an object $g$. She then generates tuple $t_{1}$, collecting information about the creation of $g$, and an encryption key $k_{1}$ to protect $t_{1}$. She then encrypts $t_{1}$ using key $k_{1}$ and stores it in the cloud. Since she is authorized to access tuple $t_{1}$, she also generates token $_{M, 1}$ enabling her to derive $k_{1}$, and hence access $t_{1}$, from her own key $k_{M}$.

Move. When a player $p_{i}$ sends an object to another player $p_{j}$, the receiver generates a tuple $t$ to keep track of her operations over the object, and a new encryption key $k$ to protect it. She then encrypts $t$ using $k$ and stores the encrypted tuple in the cloud. Since $p_{j}$ can access $t$, she also generates a token enabling her to derive $k$ from her own private key. With reference to our example, the manufacturer $M$ sends $g$ to $D$, who in turns forwards it to $R$. As a consequence of the first move action, $D$ generates tuple $t_{2}$, encrypts it with a newly chosen key $k_{2}$, and computes token token $_{D, 2}$. Similarly, as a consequence of the second move action, $R$ generates tuple $t_{3}$, encrypts it with a newly chosen key $k_{3}$, and computes token token $_{R, 3}$. The authorization and encryption policies resulting after the initialize and move steps of the protocol are illustrated in Figure 5. Note that, at this point, each tuple is visible only to its owner (i.e., the player who generated it).

Authenticate. Since each player $p_{i}$ encrypted the information that she generated with a key that only she knows and can derive, any other player $p_{j}$ who wants to access a tuple must first authenticate to the tuple owner to be granted access to the information. If player $p_{j}$ successfully authenticates with $p_{i}$ to access tuple $t$, then $p_{i}$ will grant $p_{j}$ access to $t$. Since each tuple is encrypted using a different key, to enforce such a policy change, $p_{i}$ only needs to generate a token enabling $p_{j}$ to derive the encryption key $k$ used to protect $t$ from her own private key. This guarantees that $k$ can be derived by all the users in $\operatorname{acl}(t)$, including $p_{j}$. Note that, it is not necessary to re-encrypt $t$, since simply adding the token enables $p_{j}$ to derive $k$, while not disclosing other tuples to $p_{j}$. With reference to our example, assume that $M$ would like to read $t_{3}$. To this aim, she needs to authenticate with $R$, who owns the tuple of interest, using the authentication protocol described in Section III. If the authentication is successful, $R$ grants $M$ access to $t_{3}$ and updates $\operatorname{acl}\left(t_{3}\right)=M$ to $\operatorname{acl}\left(t_{3}\right)=M R$. Since $k_{3}$ has been used to protect only tuple $t_{3}$, it is sufficient to add token token $_{M, 3}$ to the catalog to enable $M$ to access $t_{3}$. Indeed, both $M$ and $R$ will be able to derive $k_{3}$, hence $k_{3}$ will become the key of the set $\{M, R\}$ of users. Since the computation of token token $_{M, 3}$ requires knowledge of private information of both $M$ (her private key $k_{M}$ ) and of $R\left(k_{3}\right)$, the two players need to collaborate to compute it. To this aim, player $M$ uses her private key $k_{M}$ and the label of the key used to protect $t_{3}$, which is public, to compute $h\left(k_{M}, l_{3}\right)$. Then, she sends the result securely to $R$. Finally $R$ computes the token as $k_{3} \oplus h\left(k_{M}, l_{3}\right)$ and stores it in the public catalog. Figure 6 illustrates the authorization and encryption policies after the authentication of $M$ for $t_{3}$.

Even if the approach illustrated above always permits to enforce the authentication step of the protocol in Section III, this may cause a growth in the number of nodes in the key 
derivation graph. Indeed, also tuples with the same acl would be encrypted with a different key. To limit the overhead due to key management, players can use the same encryption key for different tuples when they share the same $a c l$, and dismiss keys when they are no longer used for tuple encryption. Granting or revoking access to tuples may then require to create new nodes or to delete old ones from the key derivation graph. As an example, assume that tuples $\left\{t_{1}, t_{2}, t_{3}\right\}$ belong to $M$ (e.g., they represent three different objects that left $M$ and eventually reached $D$ and $R$ ), that $D$ and $R$ were granted access to $t_{1}$, and that $D$ was granted access to $t_{2}$. Figure 7 illustrates the key derivation graph, the authorization policy, and the encryption policy for such a scenario. Assume now that, at a later time, $R$ is granted access to $t_{2}$ and hence $\operatorname{acl}\left(t_{2}\right)=M D R$. As there is already a node representing $\operatorname{acl}\left(t_{2}\right)=M D R$ in the key derivation graph, the tuple owner $M$ just needs to decrypt $t_{2}$ with $k_{M D}$ and re-encrypt it with $k_{M D R}$. After this policy update, node $M D$ becomes obsolete since there is no tuple $t$ with $\operatorname{acl}(t)=M D$. Therefore, the node and all its incoming and outgoing edges can be removed from the graph (and hence the corresponding key and tokens are removed from the encryption policy). To guarantee correct key derivation, node $M D R$ is directly connected with $M, D$ and $R$. As illustrated above, players $M$ and $R$ will then need to cooperate to compute token token $_{R, M D R}$. However, as we already have a token token $_{R, M D R}$, we need to compute token $_{D, M D R}$ instead, which requires involvement of $D$. This is inconvenient for $D$, which already had access to $t_{2}$. To avoid the cooperation of players every time the token catalog needs to be updated as a consequence of an Authenticate step, the tuple owner $p_{j}$ can agree in advance with each possible access requestor $p_{i}$ an initial key $k_{i}^{j}$, which will be used by the requestor as a starting point in the derivation process. To guarantee that each player needs to manage one secret key only, we compute such a key as $k_{i}^{j}=h\left(k_{i}, l_{j}\right)$ where $k_{i}$ is the unique private key of the requestor $p_{i}$. This way, $M$ and $D$ know $k_{D}^{M}=h\left(k_{D}, l_{M}\right), M$ and $R$ know $k_{R}^{M}=h\left(k_{R}, l_{M}\right)$. Therefore, $M$ can compute tokens token,$M D R=k_{M D R} \oplus k_{D}^{M}$ and token $_{R, M D R}=k_{M D R} \oplus k_{R}^{M}$ by herself.

\section{RELATED WORK}

The problem of enabling data owners to specify and enforce access restrictions over data stored in the cloud, without the need for the data owner to filter access requests and to trust the cloud provider for authorization enforcement, has been recently widely studied. The solutions proposed to this problem follow two different strategies: attribute based encryption (e.g., [3], [14], [26]) and selective encryption (e.g., [2], [7]-[11]). Attribute-Based Encryption (ABE) [3], [14], [26] enforces attribute based access control policies over encrypted data. To this purpose, each tuple is encrypted with a key that only the users possessing attributes that satisfy the tuple policy can derive (or vice versa). Selective encryption [10], [11] instead translates the authorization policy into an equivalent encryption policy. Intuitively, each tuple is encrypted with a key known (or that can be derived) by all and only authorized users. The solution in [11] has been extended to enforce also write privileges [8], and to support the presence of multiple data owners [9]. The problem of efficiently manage policy update operations, limiting the data owner overhead, has been also studied [2], [11]. To this aim, the proposal in [11] delegates expensive re-encryption operations to the storing server. The approach in [2] instead specifically focuses on revoke operations, and introduces a solution that does not require complete re-encryption of revoked tuples.

A line of work related to our proposal is represented by access control enforcement using RFID. An extension of attribute based access control in RFID deployments has been previously formulated in [21]. However, in contrast to the work presented in this paper, the impact of the cloud and its requirement for efficient cryptographic capabilities, as we suggest, has not been analyzed. General approaches to RFID security and privacy are extensively surveyed in [17]. An alternative access control proposals for the RFID scenario at hand is represented by [4] which is considering the problem of specifying access only to part of the object data. Effectively this is expressible by the ABAC approach. The work in [16] formulates an access control approach in which the decision maker changes when an object is passed on from one party to another party, where we are uncertain about efficiency.

When providing confidentiality in the cloud by cryptographic means, querying of encrypted data is an important topic because encryption prevents the cloud provider from directly evaluating users queries due to no access to encryption keys. Furthermore, requiring the user to download the encrypted data and locally decrypt it before processing, nullifies the benefits of outsourcing the database to the cloud. Thus, we see the line of research on processing queries directly over encrypted data through specific encryption schemes, such as [5], [15], [22], [25], as an intriguing extension to the selective access approach formulated in this work.

\section{CONCLUSIONS}

We have presented an approach based on selective encryption for enforcing downstream and upstream visibility policies in supply chain scenarios, where data about objects are stored at an external cloud service provider. The proposed approach enables companies to profitably use the sharing services offered by the cloud, while enforcing restrictions on the visibility of sensitive data. Our technique has the advantage of flexibility and enables the enforcement of arbitrary visibility policies over objects' data.

\section{ACKNOWLEDGMENT}

This work was supported by the EC within the H2020 under grant agreement 644579 (ESCUDO-CLOUD).

\section{REFERENCES}

[1] M. Atallah, M. Blanton, N. Fazio, and K. Frikken, "Dynamic and efficient key management for access hierarchies," ACM TISSEC, vol. 12, no. 3, pp. 18:1-18:43, January 2009. 
[2] E. Bacis, S. De Capitani di Vimercati, S. Foresti, S. Paraboschi, M. Rosa, and P. Samarati, "Mix\&Slice: Efficient access revocation in the cloud," in Proc. of CCS, Vienna, Austria, October 2016.

[3] J. Bethencourt, A. Sahai, and B. Waters, "Ciphertext-policy AttributeBased Encryption," in Proc. of IEEE S\&P, Oakland, CA, May 2007.

[4] D. Boneh, B. Lynn, and H. Shacham, "Short signatures from the weil pairing," Journal of Cryptology, vol. 17, no. 4, pp. 297-319, Sep 2004.

[5] A. Ceselli, E. Damiani, S. De Capitani di Vimercati, S. Jajodia, S. Paraboschi, and P. Samarati, "Modeling and assessing inference exposure in encrypted databases," ACM TISSEC, vol. 8, no. 1, pp. 119-152, 2005.

[6] S.-Y. Chou and Y. Ekawati, "Cost reduction of public transportation systems with information visibility enabled by RFID technology," in Proc. of CE, Taipei, Taiwan, July 2009.

[7] S. De Capitani di Vimercati, S. Foresti, S. Jajodia, and G. Livraga, "Enforcing subscription-based authorization policies in cloud scenarios," in Proc. of the 26th Annual IFIP WG 11.3 Working Conference on Data and Applications Security and Privacy (DBSec 2012), Paris, France, July 2012.

[8] S. De Capitani di Vimercati, S. Foresti, S. Jajodia, G. Livraga, S. Paraboschi, and P. Samarati, "Enforcing dynamic write privileges in data outsourcing," Computers \& Security, vol. 39, pp. 47-63, November 2013.

[9] S. De Capitani di Vimercati, S. Foresti, S. Jajodia, S. Paraboschi, G. Pelosi, and P. Samarati, "Encryption-based policy enforcement for cloud storage," in Proc. of SPCC, Genova, Italy, June 2010.

[10] S. De Capitani di Vimercati, S. Foresti, S. Jajodia, S. Paraboschi, and P. Samarati, "Over-encryption: Management of access control evolution on outsourced data," in Proc. of VLDB, Vienna, Austria, September 2007.

[11] — " "Encryption policies for regulating access to outsourced data," ACM TODS, vol. 35, no. 2, pp. 12:1-12:46, April 2010.

[12] B. L. Dos Santos and L. S. Smith, "RFID in the supply chain," Communications of the ACM, vol. 51, no. 10, pp. 127-131, October 2008.

[13] K. Finkenzeller, RFID Handbook: Fundamentals and Applications in Contactless Smart Cards and Identification. John Wiley \& Sons, Inc., 2003.

[14] V. Goyal, O. Pandey, A. Sahai, and B. Waters, "Attribute-based encryption for fine-grained access control of encrypted data," in Proc. of CCS, Alexandria, VA, October-November 2006.

[15] I. Hang, F. Kerschbaum, and E. Damiani, "ENKI: Access control for encrypted query processing," in Proc. of SIGMOD, Melbourne, Victoria, Australia, May - June 2015.

[16] A. Ilic, F. Michahelles, and E. Fleisch, "Dual ownership: Access management for shared item information in RFID-enabled supply chains," in Proc. of IEEE PerCom Workshops, White Plains, NY, USA, March 2007.

[17] A. Juels, "RFID security and privacy: A research survey," IEEE Journal on Selected Areas in Communications, vol. 24, no. 2, pp. 381-394, 2006.

[18] F. Kerschbaum, "Practical privacy-preserving benchmarking," in Proc. of SEC, Milan, Italy, September 2008.

[19] F. Kerschbaum, D. Dahlmeier, A. Schröpfer, and D. Biswas, "On the practical importance of communication complexity for secure multi-party computation protocols," in Proc. of SAC, Honolulu, HI, USA, March 2009.

[20] F. Kerschbaum, N. Oertel, and L. Weiss Ferreira Chaves, "Privacypreserving computation of benchmarks on item-level data using RFID," in Proc. of WiSec, Hoboken, NJ, USA, March 2010.

[21] F. Kerschbaum, "An access control model for mobile physical objects," in Proc. of SACMAT, Pittsburgh, Pennsylvania, USA, June 2010.

[22] - "Frequency-hiding order-preserving encryption," in Proc. of CCS, Denver, Colorado, USA, October 2015.

[23] C. Kuerschner, F. Thiesse, and E. Fleisch, "An analysis of data-ontag concepts in manufacturing," in Proc. of MMS, Munich, Germany, February 2008.

[24] NIST, "A survey of access control models," in Privilege (Access) Management Workshop, 2009.

[25] R. A. Popa, C. M. S. Redfield, N. Zeldovich, and H. Balakrishnan, "CryptDB: Protecting confidentiality with encrypted query processing," in Proc. of SOSP, Cascais, Portugal, October 2011.

[26] A. Sahai and B. Waters, "Fuzzy identity-based encryption," in Proc. of EUROCRYPT, Aarhus, Denmark, May 2005.

[27] S. Sarma, D. Brock, and D. W. Engels, "Radio frequency identification and the electronic product code," IEEE Micro, vol. 21, no. 6, pp. 50-54, November 2001.

[28] T. Staake, F. Thiesse, and E. Fleisch, "Extending the EPC network: the potential of RFID in anti-counterfeiting," in Proc. of SAC, Santa Fe, New Mexico, USA, March 2005.

[29] L. Weiss Ferreira Chaves and F. Kerschbaum, "Industrial privacy in RFID-based batch recalls," in Proc. of 3M4EC, Munich, Germany, September 2008. 